\title{
Atomic wavefunctions probed through strong-field light-matter interaction
}

\author{
D. Shafir ${ }^{1}$, Y. Mairesse ${ }^{2,3}$, D. M. Villeneuve ${ }^{3}$, P. B. Corkum ${ }^{3 \star}$ and N. Dudovich ${ }^{1,3 \star}$
}

\begin{abstract}
Strong-field light-matter interactions can encode the spatial properties of the electronic wavefunctions that contribute to the process ${ }^{1-4}$. In particular, the broadband harmonic spectra, measured for a series of molecular alignments, can be used to create a tomographic reconstruction of molecular orbitals ${ }^{5}$. Here, we present an extension of the tomography approach to systems that cannot be naturally aligned. We demonstrate this ability by probing the two-dimensional properties of atomic wavefunctions. By manipulating an electron-ion recollision process $^{6}$, we are able to resolve the symmetry of the atomic wavefunction with high contrast.
\end{abstract}

The basic route to spatially probe a molecular orbital involves four main steps ${ }^{5}$. First, the orbital axis is aligned in the laboratory frame ${ }^{7}$, this being accomplished by aligning the molecule. Second, an electron is ionized by a strong laser field through tunnelling ionization, after which the electron oscillates in the laser electric field and may recollide with the parent ion. Third, if the electron recollides, the recollision projects the ground state into the spatial frequencies that compose the free-electron wavefunction. The whole process, induced during less than one optical cycle, leads to the emission of extreme ultraviolet pulses with attosecond duration $^{8}$. The projected ground state is therefore obtained from the broadband spectrum of the emitted pulses. In the final step, the molecule is aligned at different angles to the recolliding electron momentum, which permits tomographic reconstruction of the orbital.

Despite its importance and generality, it has been impossible to extend this approach, known as orbital tomography, beyond simple molecular orbitals such as the $\mathrm{N}_{2}$ highest occupied molecular orbital. There are two fundamental limitations. The first arises from the coupling between ionization and recollision. When the molecule is rotated, both the tunnelling and recollision probabilities can be very strongly modulated ${ }^{9,10}$. This couples the angle dependence of tunnelling, recollision and recombination in the harmonic spectrum. These processes must be disentangled before tomography can be extended beyond sigma orbitals (where tunnelling is relatively insensitive to angle). The second limitation arises from the requirement to fix the orbital in the laboratory frame-using molecular alignment. Tomography cannot resolve degenerate orbitals that are not fixed within a molecular structure, or molecules that are difficult to align.

We overcome both limitations, generalizing tomography to atoms and by extension, to degenerate molecular orbitals. In addition, by removing the necessity to rotate the molecule, we remove the deleterious effects of tunnelling, without affecting its benefits. In fact, in the future aligning molecules will provide a new use. Alignment will enable a specific orbital to be selected for study from among a set of ionizing orbitals in complex molecules.
There are two key steps in our approach. Tunnel ionization selects the wavefunction to be probed ${ }^{11}$. Next, we manipulate the two-dimensional trajectory of free electrons. Such manipulation is achieved by adding a second-harmonic field polarized orthogonally to the fundamental field. By scanning the relative delay between the two colours, we control the angle between ionization and recollision ${ }^{12-14}$. To measure the angle, we exploit the harmonic spectrum itself. We establish that for a spherically symmetric reference atom, the angle is determined by the relative strength of the even and odd harmonics. Finally, we apply this scheme to probe the spatial properties of the $p$ state in neon atoms.

Manipulation of the free electron using a two-colour field is illustrated in Fig. 1a. The fundamental field is polarized along the $x$ axis, whereas the second-harmonic field is polarized along the $y$ axis. Tunnel ionization occurs along the instantaneous electric field direction. The free electron accelerates in the electric field and is then driven to recollide with the parent ion at an angle $\theta$. The motion of the electron is schematically described by the blue dashed line. If the ground state is spherically symmetric then the pulse emitted during each half cycle of the laser field will be polarized along the recollision direction (purple arrow).

We generate attosecond pulses with a multi-cycle pulse and therefore repeat the process at each half cycle of the laser field ${ }^{15}$. From symmetry considerations, attosecond pulses induced by the negative half cycle are polarized along the $\pi-\theta$ direction. The pulses interfere in the high-harmonic spectrum. Owing to the periodicity of the laser field, the interference can be described as:

$$
E_{n \omega_{0}} \propto E_{x} \hat{x}+E_{y} \hat{y}-\mathrm{e}^{-i n \pi}\left(E_{x} \hat{x}-E_{y} \hat{y}\right)
$$

where $n$ is the harmonic number and $E_{x}=E \cos (\theta)$ and $E_{y}=E \sin (\theta)$ are the extreme-ultraviolet field's projections along the $\hat{x}$ and $\hat{y}$ axes, respectively. Equation (1) shows that odd and even harmonics are orthogonally polarized and depend on the recollision angle according to:

$$
E_{\text {odd }}=E_{x} \hat{x}=E \cos (\theta) \hat{x} \quad E_{\text {even }}=E_{y} \hat{y}=E \sin (\theta) \hat{y}
$$

The second-harmonic field leads to symmetry breaking between adjacent half cycles. Therefore, even harmonics are polarized along the second-harmonic field polarization $\hat{y}$, whereas odd harmonics are polarized along the fundamental field polarization $\hat{x}$. Relying on the symmetry properties of the interaction, the polarization of the attosecond pulse can be analysed in a single measurement. Furthermore, as odd and even harmonics take two different

${ }^{1}$ Department of Physics of Complex Systems, Weizmann Institute of Science, Rehovot 76100, Israel, ${ }^{2}$ CELIA, Université Bordeaux I, UMR 5107 (CNRS, Bordeaux 1, CEA), 351 Cours de la Libération, 33405 Talence Cedex, France, ${ }^{3}$ National Research Council of Canada, 100 Sussex Drive, Ottawa, Ontario K1A OR6, Canada. ${ }^{*}$-mail: Paul.Corkum@nrc.ca; fniritd@wisemail.weizmann.ac.il. 
a

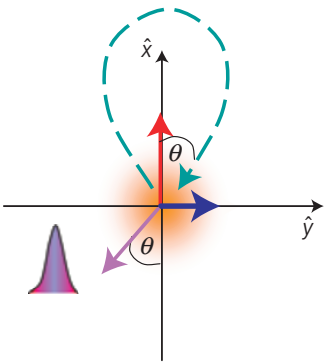

b

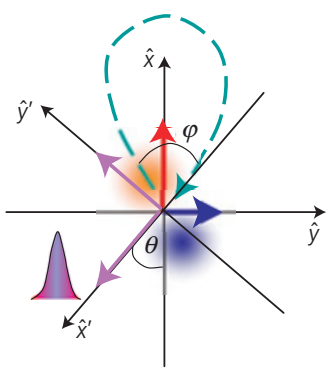

Figure 1 | Schematic diagrams of high-harmonic generation in the two colour fields. a, Spherically symmetric ground state. The red and blue arrows correspond to the fundamental and second-harmonic fields in the laboratory frame $(\hat{x}, \hat{y})$, respectively. Recollision with the ion occurs at an angle $\theta$. Extreme-ultraviolet emission is indicated by the purple arrow and is polarized along the recollision angle. $\mathbf{b}$, The recollision is induced by a $p$ state. The angle $\varphi$ is defined as the angle between ionization and recollision. The probing is read in the free electron's frame $\left(\hat{x}^{\prime}, \hat{y}^{\prime}\right)$. The extreme-ultraviolet polarization is composed of two vectorial components, polarized along $\hat{x}^{\prime}$ and $\hat{y}^{\prime}$, respectively.

projections of the electric field vector, their relative intensities reflect the recollision angle itself.

The free-electron trajectory is determined by the coherent superposition of fundamental and second-harmonic fields. As we change the two fields' delay, we modify significantly both the angle of recollision and the lateral displacement of the electron from its parent ion as it recollides. For some delays, the electron is shifted by the field such that it misses the atom. In this case, the recollision probability and therefore the emitted signal intensity are significantly reduced ${ }^{12}$. This mechanism is closely related to the reduction of the harmonic signal induced by an elliptically polarized single colour field ${ }^{16}$.

We calibrate the angle of recollision, $\theta$, by measuring high harmonics from the spherically symmetric $1 s$ state of helium atoms (see Fig. 2a). The experimental set-up is described in the Methods section. Low-signal areas (dark areas) correspond to a large displacement of the free electron from the parent ion. Owing to the symmetry breaking induced by the second-harmonic field, the spectrum contains both even and odd harmonics. A more careful examination shows that in the low-energy regime, odd harmonics disappear, whereas in the high-frequency regime even harmonics disappear. This spectral response indicates that the polarization of the high harmonics changes markedly across the harmonic spectrum. More importantly, according to equation (2), such modification results from a large variation of the recollision angle.

The range of recollision angles is controlled by the two fields' intensities and relative delay. However, we do not need to know these parameters precisely as the symmetry of the ground state enables us to measure them directly. We assume that the emitted electric field polarization changes slowly as a function of the harmonic number. Applying equation (2), we can directly extract the recollision angle. A detailed analysis is described in the Methods section. Figure $2 \mathrm{~b}$ presents the measured recollision angle as a function of the harmonic number and the two fields' delay. At the low-frequency regime, the recollision angle exceeds $60^{\circ}$. In this regime, the large values of recollision angle are accompanied by large error bars. The angle changes markedly as a function of the harmonic order and approaches zero in the high-frequency regime. According to the strong-field approximation ${ }^{17}$ (SFA), the low-frequency regime corresponds to short electron trajectories. In these trajectories, the electron is born when the fundamental field is rather low; therefore, its dynamics are dominated by the orthogonally polarized second-harmonic field. The highenergy regime, which corresponds to longer electron trajectories,
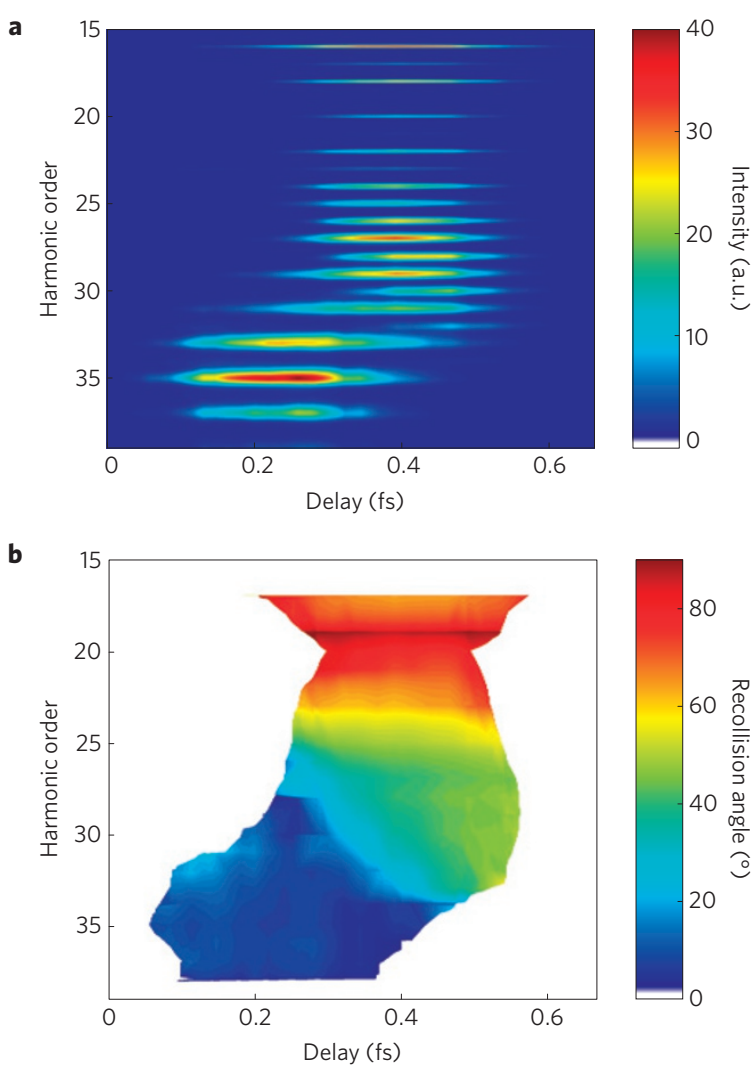

Figure 2 | Calibration of the recollision angle. a, High-harmonic spectrum from helium atoms as a function of the delay between the two colours. b, Measured electron recollision angle in the laboratory frame.

is dominated by the strong $\hat{x}$-polarized fundamental field and therefore results in low recollision angles.

At this stage, having measured the angle between the electric field direction of the fundamental beam and the recollision angle, we replace the spherically symmetric ground state by a more complex wavefunction. Although the dynamic range is limited, Fig. $2 \mathrm{~b}$ shows that we can probe the wavefunction from different angles, obtaining the information needed to identify the orbital ${ }^{1-5,13}$.

Figure $1 \mathrm{~b}$ illustrates the process for ground states that have more complex symmetries (for example, the $p$ state). Although the ionization angle did not select a unique orbital in the spherically symmetric case, it has an important role for more complex symmetries. Tunnel ionization occurs along the instantaneous electric field direction. Tunnelling theory ${ }^{18}$ suggests that in mixed $p$ states of closed-shell atoms, the state parallel to the electric field is more efficiently ionized ${ }^{11,19}$ creating an effective alignment of the atomic wavefunction. In the figure, we placed the orbital along the polarization of the field at the time of ionization and the angle $\varphi$ is defined as the angle between ionization and recollision. $\varphi$ changes with the electron's trajectory length and therefore with the harmonic number. In Fig. 2b, we have determined the recollision angle, $\theta$; however, the angle $\varphi$ is an unknown parameter in our experiment. It is uniquely related to $\theta$ in the SFA. It serves, in fact, as the effective probing angle. If the quantization axis is not modified during the optical cycle, we probe the atomic wavefunction that was selected by the tunnel ionization process. Therefore, our mechanism enables us to carry out a two-dimensional probing of the pre-selected wavefunction.

A convenient frame to read the pre-selected wavefunction is the probe's frame, or in our case, the free electron's frame. We can easily transfer the measurement from the laboratory frame to the free-electron frame by rotating the original set of axes $\hat{x}, \hat{y}$ 


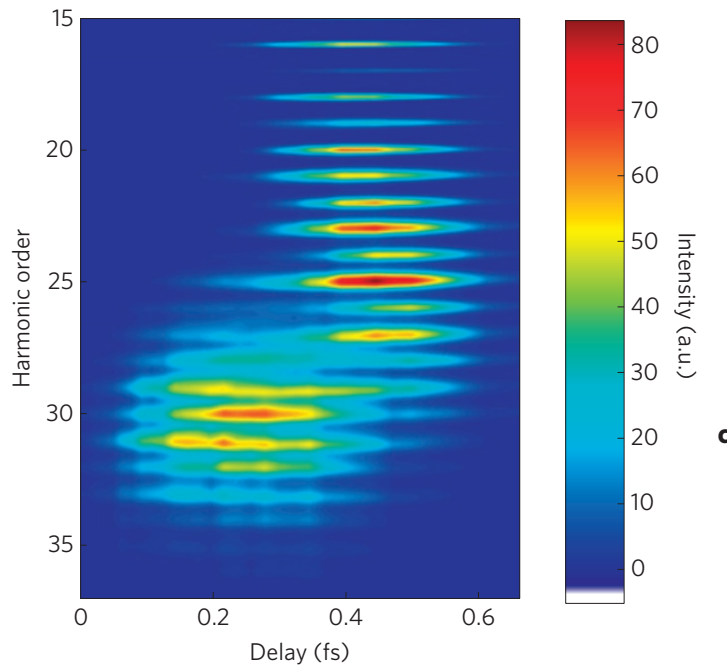

b
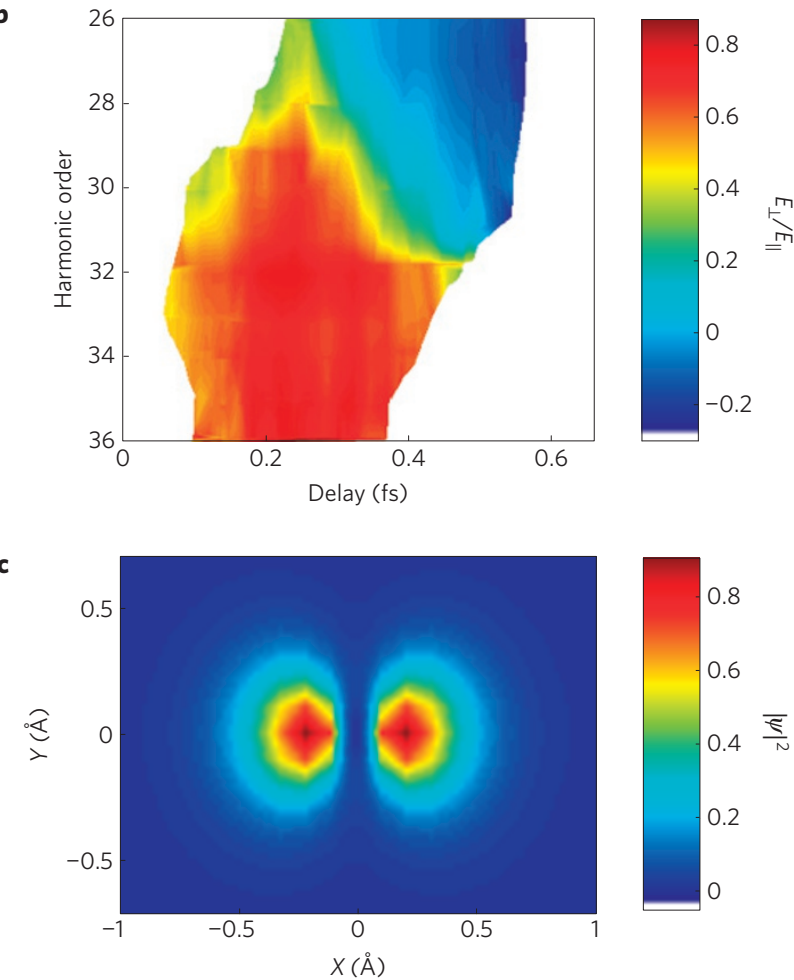

Figure 3 | Probing the atomic wavefunction in neon. a, High-harmonic spectrum from neon gas. One major difference from the helium spectrum is that here, even harmonics appear at high energies as well. $\mathbf{b}$, The ratio $E_{\perp} / E_{\|}$measured in the electron recollision frame for neon. $\mathbf{c}$, Retrieved neon mixed $2 p$ orbital $\left(|\Psi|^{2}\right)$ with a $p_{\tilde{x}}$ amplitude of 0.95 .

by an angle $\theta$, illustrated in Fig. 2. The new frame is represented in Fig. $1 \mathrm{~b}$ by the axes $\hat{x}^{\prime}$ and $\hat{y}^{\prime}$, directed along and perpendicular to the re-collision angle, respectively. The polarization of the emitted pulses is described as being composed of two components: $E_{\|}$along $\hat{x}^{\prime}$ and $E_{\perp}$ along $\hat{y}^{\prime}$. The polarization components are dictated by the two matrix elements: $\mathbf{E}_{\|} \propto\left\langle\Psi_{\mathrm{g}}(\varphi)\left|\mathbf{x}^{\prime}\right| \exp \left(\imath k_{\omega} x^{\prime}\right)\right\rangle$, $\mathbf{E}_{\perp} \propto\left\langle\Psi_{\mathrm{g}}(\varphi)\left|\mathbf{y}^{\prime}\right| \exp \left(\imath k_{\omega} x\right)\right\rangle$, where $\Psi_{\mathrm{g}}(\varphi)$ is the pre-selected ground state, aligned along $\varphi$. The vectorial properties of the emitted radiation reflect the wavefunction's symmetry. This idea has been recently demonstrated by measuring the polarization state of the high harmonics with aligned molecules ${ }^{20}$. In symmetric atomic wavefunctions, $E_{\perp} \equiv 0$. Generalizing equation (2), we describe the spectral components as:

$$
\begin{aligned}
& E_{\text {odd }}(\omega)=-E_{\|} \cos (\theta)+E_{\perp} \sin (\theta) \\
& E_{\text {even }}(\omega)=-E_{\|} \sin (\theta)-E_{\perp} \cos (\theta)
\end{aligned}
$$

where both even and odd harmonics contain a coherent addition of the two polarization components.

A natural candidate that lacks inversion symmetry is neon with its highest occupied orbital being a $2 p$ state. Figure 3 a describes the high-harmonic spectrum as a function of the two colours' delay. As in the helium experiment (see Fig. 2), we measure both odd and even harmonics. Comparing the two measurements, we observe a striking difference. Whereas even harmonics measured from helium have disappeared in the high-frequency regime, in the neon measurement they are as strong as the odd harmonics.

The neon spectrum was separated into odd- and even-harmonic spectra and interpolated. We now rely on the recollision angle calibration $(\theta)$ to transfer the measurement from the laboratory frame $(\hat{x}, \hat{y})$ to the recolliding electron's frame $\left(\hat{x}^{\prime}, \hat{y}^{\prime}\right)$. The relative signs of $E_{\text {odd }}$ and $E_{\text {even }}$ were chosen by theoretical considerations. Each point in the neon even/odd spectrum was rotated by the corresponding angle. The frequency scale of the $\theta$ measurement was shifted by $3.03 \mathrm{eV}$ to compensate for differences in ionization potentials. Using equation (3), we extract the ratio $E_{\perp} / E_{\|}$. Figure $3 \mathrm{~b}$ presents the analysed ratio, as a function of the harmonic number and the two fields' delay.

The extracted ratio reflects the symmetry of the probed wavefunction as seen by the free electron. This is equivalent to the polarization rotation observed with aligned molecules ${ }^{20}$. There are two conditions required to probe symmetries that are more complex than the spherically symmetric one. The first requires that tunnel ionization selects a preferable quantization axis ${ }^{11}$. The second is a large angle between the quantization axis and the probing direction. The measured ratio approaches one in the high-frequency range, which indicates that these two conditions are met in this regime. The first is reached by selective ionization, and the second through manipulation of the free-electron trajectory ${ }^{14}$. Semiclassical simulation shows that in this spectral regime, large $\varphi$ values are indeed obtained.

Finally, we analysed the measured ratio $E_{\perp} / E_{\|}$to resolve the selected orbital. Specifically, we compared the measured symmetry with the theoretically calculated one to resolve the degree of selectivity. Using a fitting algorithm, we extracted the relative population of each of the three orthogonal $p$ states. A detailed analysis is described in the Methods section. The wavefunction was found to be almost a pure aligned state, with a relative amplitude weight of $0.95 \pm 0.05$. This measurement agrees with tunnelling theory, which predicts a relative amplitude weight of 0.98 (ref. 18). Figure $3 \mathrm{c}$ describes the density function of the selected orbital.

We have demonstrated a new concept in orbital tomographythe electronic structure is probed in a correlated manner. In our experiment, the electronic wavefunction is not well defined in the laboratory frame. Its quantization axis rotates within the optical cycle, following the instantaneous direction of the electric field. However, the electronic wavefunction is strongly correlated with the 
free electron and therefore well defined in its frame. In other words, we measure a field-selected wavefunction, which provides a direct insight into the outcome of tunnelling ionization-one of the most fundamental processes in strong-field light-matter interactions. We have demonstrated that the probed wavefunction is selected by the ionization step and preserves its orientation during the optical cycle.

In our experiment, the range of probing angles was limited. However, interchanging the role of the fundamental and secondharmonic fields enables all probing angles-for example, a strong $400 \mathrm{~nm}$ field combined with a weaker $800 \mathrm{~nm}$ field. With sufficient angular information, tomographic reconstruction of atomic orbitals will be achieved.

Although this letter focuses on the measurements of atomic wavefunctions, our approach will have a significant impact on molecular tomography. First, previously inaccessible orbitals, such as degenerate states or orbitals in molecules with small polarizability can now be probed. Second, as it is no longer necessary to rotate the molecules relative to the ionizing field, we decouple tunnelling and recombination. Alignment can now serve a new and even more valuable purpose than before. We can use the sensitivity of tunnelling and recollision to select any orbital that preferentially ionizes at a specific alignment angle. For example, in $\mathrm{CO}_{2}$ the $\Sigma_{\mathrm{g}}$ orbital preferentially ionizes at $45^{\circ}$ (ref. 9), the $\Pi_{\mathrm{u}}$ orbital at $90^{\circ}$ and the $\Sigma_{\mathrm{u}}$ orbital at $0^{\circ}$. Once selected, with our two-colour approach, each could be individually imaged. Finally, if ionization preferentially selects an orientation in heteronuclear molecules ${ }^{21}$, then these orbitals can be imaged without orienting the molecule. As orbital tomography is extended to a wide range of systems, the potential for dynamic imaging that is inherent in the technology becomes increasingly significant.

Our approach is not limited to the measurement of static wavefunctions. The high temporal resolution provided by the free electron can be combined with the spatial properties of the measurement. Dynamics that occurs between ionization and recollision will be probed by the free electron with sub-cycle resolution. Such dynamics include for example, spin-orbit coupling ${ }^{22}$, sub-cycle Stark shifts or multi-electron dynamics in molecules ${ }^{23}$.

\section{Methods}

Experimental methods. High harmonics are generated with $30 \mathrm{fs}, 50 \mathrm{~Hz}, 2 \mathrm{~mJ}$, $800 \mathrm{~nm}$ laser pulses in an atomic gas jet. We estimated the pulse intensity to be $1.8 \times 10^{14} \mathrm{~W} \mathrm{~cm}^{-2}$ according to the cutoff harmonic. The second-harmonic field (on a $25 \%$ intensity level) is produced using a $100 \mu \mathrm{m}$ type- $\mathrm{I} \mathrm{BaB}_{2} \mathrm{O}_{4}$ crystal. The second-harmonic field is orthogonally polarized with respect to the fundamental field. Group-velocity dispersion is compensated using a birefringent crystal (calcite). The phase of the second-harmonic field relative to the fundamental field is controlled with $250 \mu \mathrm{m}$ of BK7 glass. High harmonics are generated by focusing the two beams into a pulsed gas jet. The harmonic spectrum is measured by an extreme-ultraviolet spectrometer. The experimental set-up is described in more detail in ref. 24.

Propagation effects can have an important role in this experiment, as in many other experiments that use the free electron as a temporal or spatial probe ${ }^{1-5,20}$. These effects can be minimized by carefully choosing the focal parameters (see a detailed analysis in ref. 25). We minimize propagation effects by choosing the focal parameters such that the jet length $(\sim 1 \mathrm{~mm})$ is short compared with the Rayleigh length $(\sim 2 \mathrm{~cm})$. Furthermore, our approach provides a robust measurement of the free electron's dynamics. The measurement is based on the ratio between even to odd harmonics rather than the absolute signal. By measuring the ratio, we eliminate the sensitivity of the experiment to different parameters such as the detector efficiency or propagation effects.

Extracting the recollision angle. To extract the recollision angle, we assume that in the absence of electronic resonances the dipole moment changes slowly as a function of the harmonic number. On the basis of this assumption, odd and even harmonics were separated and the area between adjacent harmonics was interpolated. We corrected the measured spectra according to the spectrometer efficiencies for the two polarizations. Using equation (2) and relying on the slow variation of the dipole moment, we can directly extract the recollision angle:

$$
\tan (\theta)(n, \tau)=\sqrt{\frac{I_{\text {even }}(n, \tau)}{I_{\text {odd }}(n, \tau)}}
$$

where $I_{\text {even }}$ and $I_{\text {odd }}$ are the even- and odd-harmonic intensities respectively, $n$ is the harmonic order and $\tau$ is the two colours' delay. This analysis provides an independent measurement of the recollision angle that does not rely on theoretical modelling of the free electron's dynamics.

Resolving the atomic wavefunction. We assume that the atomic ground state in neon is composed of three orthogonal $p$ states

$$
\psi_{\mathrm{g}}(x, y, z)=\epsilon_{\bar{x}} p_{\bar{x}}+\epsilon_{\bar{y}} p_{\bar{y}}+\epsilon_{\bar{z}} p_{\bar{z}}
$$

where $\tilde{z}$ is the propagation axis of the laser beam, $\tilde{x}$ and $\tilde{y}$ are directed along and perpendicular to the ionization axis respectively, and $\epsilon_{\bar{x}, \bar{y}, \bar{z}}$ are the amplitude coefficients of each of the $p$ states. The free electron recollides with $p_{\bar{z}}$ with an angle of $90^{\circ}$. As it is symmetric along the $\tilde{z}$ axis, its overlap with the antisymmetric $p_{\bar{z}}$ wavefunction cancels out and does not contribute to the harmonics signal. $p_{\bar{y}}$ and $p_{\bar{z}}$ are quantized orthogonally to the ionization axis and we therefore assume that they carry the same weight. Our reconstruction procedure resolves a single parameter defined as: $s \equiv \epsilon_{\bar{y}} / \epsilon_{\bar{x}}$. Pure selectivity corresponds to $s=0$, whereas zero selectivity corresponds to $s=1$.

In Fig. $3 b$, we present the measured ratio $E_{\perp} / E_{\|}$. This ratio reflects the symmetry of the probed wavefunction and therefore also of the population ratio $s$. We resolve $s$ by comparing our experiment with a theoretical calculation. We calculate the ratio $E_{\perp} / E_{\|}$theoretically as a function of $s$. The atomic wavefunctions were calculated using a standard Hartree-Fock $a b$ initio program GAMESS (ref. 26). The probing angles $(\varphi)$ were calculated according to classical electron's trajectories using SFA. The parameters of the experiment such as the two fields' delay and the relative fields' intensities are calibrated by the helium experiment presented in Fig. 2. The two dipole components are determined according to: $\mathbf{E}_{\|} \propto\left\langle\Psi_{\mathrm{g}}(\varphi, s)\left|\mathbf{x}^{\prime}\right| \exp \left(\imath k_{\omega} x^{\prime}\right)\right\rangle, \mathbf{E}_{\|} \propto\left\langle\Psi_{\mathrm{g}}(\varphi, s)\left|\mathbf{x}^{\prime}\right| \exp \left(\imath k_{\omega} x^{\prime}\right)\right\rangle$. The ratio $R(s, \tau, n) \equiv E_{\perp} / E_{\|}$is evaluated as a function of the population ratio $s$, the two fields' delay $\tau$ and the harmonic number $n$. The comparison between theory and experiment is expressed as:

$$
\Delta(s)=\sum_{\tau} \sum_{n}\left|R(s, \tau, n)_{\text {theory }}-R(\tau, n)_{\text {experiment }}\right|
$$

$\Delta(s)$ represents the integrated difference between a theoretical calculation, based on selectivity $s$, and the experimental results. Finally, we extract $s$ by minimizing $\Delta(s)$ with respect to $s$ and find that $s=0.22 \pm 0.15$, that is, $\epsilon_{\bar{x}}=0.95, \epsilon_{\bar{y}}=\epsilon_{\bar{z}}=0.21$. We conclude that the wavefunction is almost a pure aligned state, which can be expressed as $\left|\Psi_{\mathrm{g}}(x, y, z)\right|^{2}=\left|0.95 \times p_{\bar{x}}\right|^{2}+\left|0.21 \times p_{\bar{y}}\right|^{2}+\left|0.21 \times p_{\bar{z}}\right|^{2}$.

\section{Received 22 January 2008; accepted 18 March 2009;} published online 26 April 2009

\section{References}

1. Lein, M., Hay, N., Velotta, R., Marangos, J. P. \& Knight, P. L. Role of the intramolecular phase in high-harmonic generation. Phys. Rev. Lett. 88 183903 (2002).

2. Vozzi, C. et al. Controlling two-center interference in molecular high harmonic generation. Phys. Rev. Lett. 95, 153902 (2005).

3. Kanai, T., Minemoto, S. \& Sakai, H. Quantum interference during high-order harmonic generation from aligned molecules. Nature 435, 470-474 (2005).

4. Torres, R. et al. Probing orbital structure of polyatomic molecules by high-order harmonic generation. Phys. Rev. Lett. 98, 203007 (2007).

5. Itatani, J. et al. Tomographic imaging of molecular orbitals. Nature 432, 867-871 (2004).

6. Corkum, P. B. Plasma perspective on strong field multiphoton ionization. Phys. Rev. Lett. 71, 1994-1997 (1993).

7. Stapelfeldt, H. \& Seideman, T. Aligning molecules with strong laser pulses. Rev. Mod. Phys. 75, 543-557 (2003).

8. Corkum, P. B. \& Krausz, F. Attosecond science. Nature Phys. 3, 381-387 (2007).

9. Pavicic, D. et al. Direct measurement of the angular dependence of ionization for $\mathrm{N}_{2}, \mathrm{O}_{2}$, and $\mathrm{CO}_{2}$ in intense laser fields. Phys. Rev. Lett. 98, 243001 (2007).

10. Meckel, M. et al. Laser induced electron tunneling and diffraction. Science 320, 1478-1482 (2008)

11. Young, L. et al. X-ray microprobe of orbital alignment in strong-field ionized atoms. Phys. Rev. Lett. 97, 083601 (2006).

12. Kim, I. J. et al. Highly efficient high-harmonic generation in an orthogonally polarized two-color laser field. Phys. Rev. Lett. 94, 243901 (2005).

13. Kitzler, M. \& Lezius, M. Spatial control of recollision wave packets with attosecond precision. Phys. Rev. Lett. 95, 253001 (2005).

14. Kitzler, M., Xie, X., Scrinzi, A. \& Baltuska, A. Optical attosecond mapping by polarization selective detection. Phys. Rev. A 76, 011801 (2007).

15. Antoine, P., L'Huillier, A. \& Lewenstein, M. Attosecond pulse trains using high order harmonics. Phys. Rev. Lett. 77, 1234-1237 (1996). 
16. Budil, K. S., Salières, P., Perry, M. D. \& L'Huillier, A. Influence of ellipticity on harmonic generation. Phys. Rev. A 48, R3437-R3440 (1993).

17. Lewenstein, M., Balcou, P., Ivanov, M. Y., L’Huillier, A. \& Corkum, P. B. Theory of high-harmonic generation by low-frequency laser fields. Phys. Rev. A 49, 2117-2132 (1994).

18. Ammosov, M. V., Delone, N. B. \& Krainov, V. P. Tunnel ionization of complex atoms and of atomic ions in an alternating electromagnetic field. Sov. Phys. JETP 64, 1191-1194 (1986).

19. Otobe, T., Yabana, K. \& Iwata, J. I. First-principles calculations for the tunnel ionization rate of atoms and molecules. Phys. Rev. A 69, 053404 (2004).

20. Levesque, J. et al. Polarization state of high-order harmonic emission from aligned molecules. Phys. Rev. Lett. 99, 243001 (2007).

21. Bandrauk, A. D. \& Kamta, G. L. Phase dependence of enhanced ionization in asymmetric molecules. Phys. Rev. Lett. 94, 203003 (2005).

22. Santra, R., Dunford, R. W. \& Young, L. Spin-orbit effect on strong-field ionization of krypton. Phys. Rev. A 74, 043403 (2006).
23. Lezius, M. et al. Nonadiabatic multielectron dynamics in strong field molecular ionization. Phys. Rev. Lett. 86, 51-54 (2001).

24. Dudovich, N. et al. Measuring and controlling the birth of attosecond XUV pulses. Nature Phys. 2, 781-786 (2006).

25. Levesque, J. et al. High harmonic generation and the role of atomic orbital wave functions. Phys. Rev. Lett. 98, 183903 (2007).

26. Schmidt, M. W. et al. General atomic and molecular electronic structure system. J. Comput. Chem. 14, 1347-1363 (1993).

\section{Acknowledgements}

We thank A. Shiner, C. Trallero, N. Kajumba and F. Légaré for carrying out pressure scan experiments to show that phase mismatch effects were negligible.

\section{Additional information}

Reprints and permissions information is available online at http://npg.nature.com/ reprintsandpermissions. Correspondence and requests for materials should be addressed to N.D. or P.B.C. 